\title{
Mobility And Energy Extensions For The IEEE 802.15.4 Standard
}

\author{
Imed Romdhani, Ahmed Y. Al-Dubai, *Wael Guibene \\ Edinburgh Napier University, School of Computing, 10 Colinton Road, Edinburgh EH10 5DT, UK \\ * Intel Labs, Collinstown Industrial Park, Leixlip, R148, Co. Kildare, Ireland \\ Email: \{I.Romdhani; A. Al-Dubai\}@napier.ac.uk \\ *Email:Wael.Guibene@intel.com
}

\begin{abstract}
The IEEE 802.15.4 standard is designed for low-power and lowrate wireless Personal Area Networks. Although energy is a key parameter in shaping the communication and interaction methods of wireless sensor nodes, the specification of the MAC layer doesn't embed enough information for energy and mobility support. For instance, using the IEEE 802.15.4 beacon frames, a node will be able just to discover whether other peers are powered by mains or are they fully or reduced functional devices. However, sensors nodes are able to gain power by different means including: rechargeable batteries, moving sensor charger, ambient or renewable energy, or a combination of these methods. To address these issues, we propose an extension of the IEEE 802.15.4 frame formats to support mobility and embed new energy powering modes and levels. We discuss how the MAC layer extensions could be used to optimize device discovery and routing processes in IEEE 802.15.4 based wireless sensor networks.
\end{abstract}

\section{Categories and Subject Descriptors}

C.2.2 [Network Protocols]: Link-layer protocols; Mobile networks.

\section{General Terms}

Design, Performance, Experimentation.

\section{Keywords}

IEEE 802.15.4, Wireless Sensor Networks, Internet of Things, Mobility.

\section{INTRODUCTION}

The IEEE 802.15.4 different standard versions are designed for different types of low-power and low-rate wireless Personal Area Networks [1]. Tiny wireless sensors implementing these standards are designed to operate typically in large-scale and lowpower wireless industrial applications including smart transport, eHealth, oil and gas surveillance and environment monitoring. These sensor nodes are required sometimes to cover geographically

Permission to make digital or hard copies of all or part of this work for personal or classroom use is granted without fee provided that copies are not made or distributed for profit or commercial advantage and that copies bear this notice and the full citation on the first page. Copyrights for components of this work owned by others than ACM must be honored. Abstracting with credit is permitted. To copy otherwise, or republish, to post on servers or to redistribute to lists, requires prior specific permission and/or a fee. Request permissions from Permissions@acm.org.

MoMM 2015, December 10-12, 2015, Brussels, Belgium

(C) 2015 ACM. ISBN 978-1-4503-3493-8/15/12 .. \$15.00

DOI: http://dx.doi.org/10.1145/2837126.2837167 widespread indoor and outdoor spaces and therefore, deploying mobile or renewable-energy based sensors is a challenging deployment issue. Different solutions have been proposed in the literature to address the network lifetime elongation problem in wireless chargeable and non-chargeable sensor networks. Energy conservation, ambient energy harvesting, incremental deployment, battery replacement, and wireless charger are among the typical suggested solutions. However, the majority of these solutions didn't revisit the standard specification to implement the new proposed techniques. Although such approach helps to keep the standard simple, the proposed solutions generally introduce an extra processing overhead at the upper layers and complicate the management and maintenance tasks for wireless sensor networks. Indeed, embedding few changes in the core of the standard itself will make it more flexible and robust to accommodate enhanced energy conserving techniques and optimize at the same time linklayer association and IP routing processes. To achieve that, this paper presents new IEEE 80.2.15.4 frame formats to embed mobility capability and integrate new power charging modes.

The rest of this paper is structured as follows. Section II presents a critical review of the related work. Section III describes the new MAC layer extensions and how to implement them using the existing standard specification of IEEE 802.15 .4 protocol. Finally the conclusion and future work are discussed in Section IV.

\section{RELATED WORK}

The IEEE 802.15.4 is a standard designed for low-power, low data rate wireless communication between small devices. It defines primarily two types of devices: Full Function Device (FFD) and Reduced Function Device (RFD). An FFD is a device that is capable to talk to all types of devices and it performs link-layer routing (mesh-under routing), whereas an FFD device can just talk to an FFD and it has less power and processing capabilities compared to an FFD. To form a topology (star, cluster tree based, or mesh), all devices are segregated into one or many Personal Area Networks (PAN). Consequently, devices will be able to communicate between PANs (inter-PAN) or within their own PAN (intra-PAN). For each PAN, a PAN coordinator is elected during the association process using a combination of four types of linklayer frames: beacon, command (association, disassociation, data beacon requests, conflict notification), data, and acknowledgement. According to current standard specification, the PAN coordinator (FFD device) will often be mains-powered, while the other devices will most likely be battery powered. An intended coordinator could not be a full operating coordinator before following a waiting procedure. In addition, nodes could not be effectively associated to a coordinator (despite receiving an association confirmation) before receiving their designated guaranteed time slot (GTS). 
During these delays, the association decision could be further optimized if the nodes could filter and classify the power and the mobility capabilities of each intended coordinator. Alternatively, the coordinator can adjust GTS allocation based on the mobility and energy capabilities of neighboring nodes. Although the power consumption is of significant concern, the IEEE 802.15.4 standard was developed with limited power supply availability in mind [1]. Additional power management considerations are kept beyond the scope of the standard specification, which opened the door for many new extensions.

Authors in [2] proposed an extensive and critical review of existing solutions that attempted to improve the 802.15.4 MAC protocol. These solutions are categorized into 8 approaches: parameter tuning-based, cross layer-based, IEEE 802.11-based, priority-based, duty cycle-based, backoff-based, QoS-based (GTS oriented), and hidden terminal resolution-based. Within these approaches, QoS-based and parameter tunining-based approaches are among the proposed solutions that attempted to modify the standard specification to implement new algorithms. Following this approach, authors in [3] and [4] proposed two new re-association procedures to deal with nodes mobility in IEEE 802.15.4 WSNs. In the first approach, the handover process is triggered by parent nodes based on their residual energy whereas in the second approach, the handover process is triggered by mobile nodes upon location change. To implement these new procedures, new fast association frames are designed. Similar implementation approach is also proposed by [5]. Authors in [6] have introduced two different and adaptive beacon frame formats for source and receiver sensing nodes. In their priority-based MAC (EEP-MAC) protocol for energy harvesting, the authors introduced a source beacon that contains a new priority flag to differentiate between normal and priority date. For the receiver beacon frame a new energy state flag is embedded along with other fields. Instead of handling the energy and the mobility using MAC layer extensions, authors in [7] proposed a multicontext management fuzzy-based decision method that they integrated within IEEE 802.15.4 standard scope to handle mobility. This mobility and module decision processes adapt in real-time the intra-cluster communications depending on the detected local and temporal context patterns (i.e. in terms of nodes activity, mobility, energy autonomy, etc.) and triggers a handover mechanism to ensure the stability of the entire wireless sensor network. Similarly, authors in [8] and [9] addressed the mobility issue from a cross-layer perspective. In [8], the authors embedded the location of the mobile nodes in the routing operation and then utilized this information by the MAC layer transmission power control to adjust the transmission range of the node. More other solutions that address the mobility issues and its relationship with energy consumption are compared and contrasted in [10] while [11] presents a comprehensive review of existing solutions that cover all OSI layers.

Although there are different approaches to address the mobility and the energy issues in IEEE 802.15.4 based WSN, we do believe that a simple extension of the MAC layer of this standard will facilitate the implementation of the above described solutions and will not compromise the simplicity of the standard itself. To this end, we propose new IEEE 802.15.4 MAC frame formats to embed new mobility and energy charging capabilities. The new extensions will ease mobility management and enhance the association and the routing processes.

\section{PROPOSED SOLUTION}

To embed various power charging capabilities and integrate the mobility feature in IEEE 802.15.4 MAC layer specification, we propose to extend the format of some MAC layer frames to enhance the discovery, the association and the routing processes in hybrid wireless sensor networks.

\subsection{Energy Extention}

The IEEE 802.15.4 standard was developed with limited power supply availability in mind. To deploy and implement this standard, additional power management considerations should be introduced. Within this context, we propose to extend the IEEE 802.15.4 frame formats to embed new classification of energy modes, current energy levels and mobility capability. We introduce three new fields in the frame header to code different power modes and add for each associated mode the corresponding classification level of the remaining residual energy. The new fields which are coded in the capacity information field of the frame header are: Power Mode (PM), Power Level (PL) and Mobility Flag (Mob). As illustrated by Figure 1, the PM filed is a 3-bit long field and it includes 8 different power charging modes: mains powered, powered with rechargeable battery (ambient energy harvesting), powered with non-rechargeable battery, powered with wireless charging sensor, hybrid powered, and reserved values. Compared to the specification of the RPL routing protocol where the Node Energy Object is a 2-bit field encoding four types of power sources in total (including one reserved for future use), our solution encompasses a wide variety of power sources (Eight modes). By encompassing this information in the link-layer, cross-layer protocol design for wireless sensor networks would be facilitated and exchange control information between protocol layers for context-aware application would be possible. One particular possibility would be the design of unified Layer 2 (L2) abstractions for Layer 3 (L3)-driven fast handovers for mobile Internet of Things as it was introduced for basic Mobile IP based protocols [12]. Figure 2 illustrates the message sequence chart for the association procedure using the new extended frames.

A: Standard Capability Information field format :
\begin{tabular}{|c|c|c|c|c|c|c|}
\hline Bits: 0 & $\mathbf{1}$ & $\mathbf{2}$ & $\mathbf{3}$ & $\mathbf{4 - 5}$ & $\mathbf{6}$ & $\mathbf{7}$ \\
\hline Reserved & $\begin{array}{l}\text { Device } \\
\text { Type }\end{array}$ & $\begin{array}{l}\text { Power } \\
\text { Source }\end{array}$ & $\begin{array}{l}\text { Receiver } \\
\text { On When } \\
\text { Idle }\end{array}$ & Reserved & $\begin{array}{l}\text { Security } \\
\text { Capability }\end{array}$ & $\begin{array}{l}\text { Allocate } \\
\text { Address }\end{array}$ \\
\hline
\end{tabular}

B : Extended Capability Information field format :

\begin{tabular}{|c|c|c|c|c|c|c|c|c|}
\hline Bits: $\mathbf{0}$ & $\mathbf{1}$ & $\mathbf{2 - 4}$ & $\mathbf{5 - 6}$ & $\mathbf{7}$ & $\mathbf{7}$ & $\mathbf{8}$ & $\mathbf{9}$ & $\mathbf{1 0 - 1 5}$ \\
\hline $\begin{array}{l}\text { Version of Cl } \\
\begin{array}{l}\text { Device } \\
\text { Type }\end{array}\end{array}$ & $\begin{array}{l}\text { Power } \\
\text { Mode }\end{array}$ & $\begin{array}{l}\text { Power } \\
\text { Level }\end{array}$ & $\begin{array}{l}\text { Mobility } \\
\text { Flag }\end{array}$ & $\begin{array}{l}\text { Security } \\
\text { Capability }\end{array}$ & $\begin{array}{l}\text { Receiver } \\
\text { On } \\
\text { When } \\
\text { Idle }\end{array}$ & $\begin{array}{l}\text { Allocate } \\
\text { Address }\end{array}$ & Reserved \\
$\qquad \begin{array}{l}\text { Version of } \mathrm{Cl}=0 \text { : Standard Capability Information field format (8 bits in total) } \\
\text { Version of } \mathrm{Cl}=1 \text { : Extended Capability Information field format (16 bits in total) }\end{array}$ \\
\hline
\end{tabular}

Figure 1. Extended capability information field format.

For each type of charging mode, we also indicate the current level or status of the remaining residual power. Four new levels are introduced: high, medium, low and critical. An implementation may choose to adjust the level threshold intervals based on the specification of the application and the hardware used. 


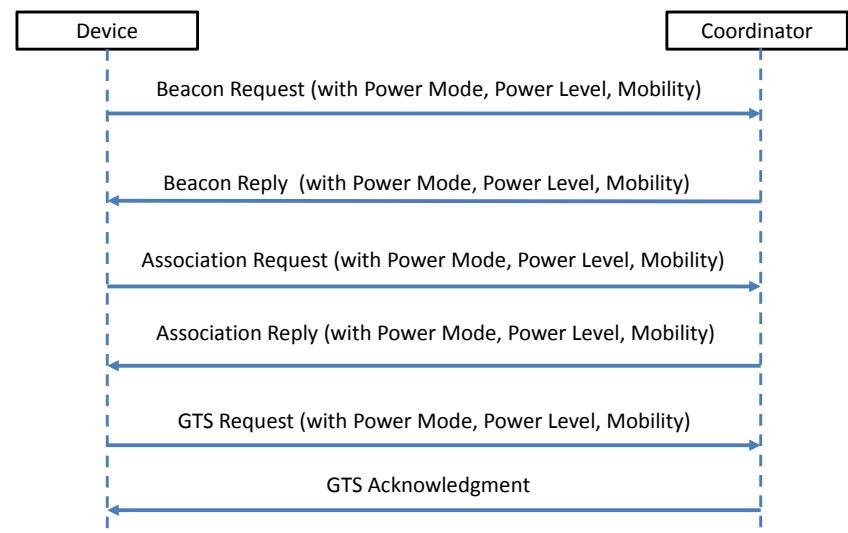

Figure 2. Message sequence chart for new association method.

Table 1. Power Mode field

\begin{tabular}{|l|l|}
\hline Power Mode & Description \\
\hline 000 & Mains powered \\
\hline 001 & Powered with rechargeable battery. \\
\hline 010 & $\begin{array}{l}\text { Powered with non-rechargeable } \\
\text { (replacement) battery. }\end{array}$ \\
\hline 011 & $\begin{array}{l}\text { Powered with stationary wireless charging } \\
\text { sensor. }\end{array}$ \\
\hline 100 & $\begin{array}{l}\text { Powered with mobile wireless charging } \\
\text { sensor. }\end{array}$ \\
\hline 101 & $\begin{array}{l}\text { Powered with a combination of methods: } \\
\text { primary storage, energy scavenging, and } \\
\text { secondary storage. }\end{array}$ \\
\hline 110 & $\begin{array}{l}\text { Node can provide power energy to other } \\
\text { nodes. }\end{array}$ \\
\hline 111 & Reserved for future use. \\
\hline
\end{tabular}

Table 2. Power Level field

\begin{tabular}{|l|l|}
\hline Power Level & Indicative residual battery level in \% \\
\hline High & Above $60 \%$ of the full battery capacity. \\
\hline Medium & $\begin{array}{l}\text { Between } 40 \text { and } 60 \% \text { of the full battery } \\
\text { capacity. }\end{array}$ \\
\hline Low & $\begin{array}{l}\text { Between } 20 \text { and } 40 \% \text { of the full battery } \\
\text { capacity. }\end{array}$ \\
\hline Critical & Less than $20 \%$ of the full battery capacity \\
\hline
\end{tabular}

\subsection{Mobility Extention}

While a traditional wireless sensor network is mainly composed of stationary nodes, new industrial applications require using mobile nodes either to recharge nodes which are suffering from battery depletion or to collect data from saturated sinks. When mobile nodes interact with their neighbors, they will not trigger just topology changes but they will also change data traffic patterns. The detection of new nodes (mobile or stationary) usually takes place at the link-layer before being reflected at the upper routing and transport layers. Therefore it would be more reasonable to include the mobility capability of sensor nodes at the link-layer before propagating it into upper OSI layers. For this reason, we introduce a new mobility flag into the beacon, association and GTS frames of the IEEE 802.15.4 standard. When the mobility flag is included, the PAN coordinator or a coordinator for example will indicate its presence and its mobility capability on a PAN to other devices. This allows other devices to perform device discovery and choose which coordinator they will prefer to join. When a mobile wireless charger is introduced in an existing wireless sensor network, nodes will probably need to join and associate temporary with the mobile PAN coordinator before switching back to the old stationary coordinator. The handover between the two coordinators could be seamlessly managed but this issue is out of the scope of this present work. The mobility feature will also allow a PAN coordinator or a coordinator to effectively manage its resources and distribute them among mobile and stationary devices before confirming definitely an association decision (within macResponseWaitTime interval time). A PAN coordinator may implement an association policy that balances between mobile and stationary devices. A typical scenario would be setting a fixed threshold for mobile sensor devices for a PAN. Depending on the number of mobile devices allowed to associate with a PAN coordinator or a given coordinator, this latter may also implement a GTS allocation and management policy that allocate more or longer GTS timer slots to the mobile devices to overcome the handover issues or to prioritize for example mobile sinks for certain types of critical applications.

In brief, by extending the IEEE 802.15.4 MAC frame formats and introducing new fields for energy powering and consumption levels and for mobility support, the proposed solution open widely the horizons to implement several QoS-based and cross-layer solutions proposed in the literature that were implemented with impractical and hard to deploy methods. New intelligent methods could easily exploit the proposed extensions to optimize data delivery, fast mobility and energy conservation techniques in wireless sensor networks. Mobile robots used in sensing fields would be easy to deploy and their routing protocols will be optimized.

\section{CONCLUSION}

In this paper we proposed few simple modifications to the IEEE 802.15.4 beacon, association and GTS frames to embed both mobility and power charging capabilities. The new extensions will ease the deployment of hybrid wireless sensor networks and facilitate the standardization of layer 2 and layer 3 primitives for fast handover and cross-layer design. Despite its theoretical clear advantages, the proposed solution needs to be validated by extensive simulations and real experimentation, which we target to realize in future works in collaboration with world leading Internet of Things research labs..

\section{REFERENCES}

[1] IEEE Computer Society, "IEEE Standard for Local and metropolitan area networks, Part 15.4: Low-Rate Wireless Personal Area Networks (LR-WPANs) : IEEE Std 802.15.4TM-2011", Page(s):1 - 314, E-ISBN : 978-07381-6683-4, 05 September 2011.

[2] Khanafer, M.; Guennoun, M.; Mouftah, H.T., "A Survey of Beacon-Enabled IEEE 802.15.4 MAC Protocols in Wireless Sensor Networks", IEEE Communications Surveys \& Tutorials, Volume: 16, Issue: 2, Pages: 856 876, 2014.

[3] Zayneb Trabelsi Ayoub, Sofiane Ouni, "New Reassociation Procedures for Reliable Handover in IEEE 
802.15.4 Wireless Sensor Networks", Ad Hoc Networks Lecture Notes of the Institute for Computer Sciences, Social Informatics and Telecommunications Engineering, Volume 129, 2014, pp 3-14, Jan 2014.

[4] Zayneb Trabelsi Ayoub, Sofiane Ouni, “A Fast Handover Procedure Based on Smart Association Decision for Mobile IEEE 802.15.4 Wireless Sensor Networks", Wireless Internet Lecture Notes of the Institute for Computer Sciences, Social Informatics and Telecommunications Engineering, Volume 146, 2015, pp 1-7, May 2015.

[5] Javed, M.; Zen, K.; Bin Lenando, H.; Zen, H, " Fast association process (FAP) of beacon enabled for IEEE 802.15.4 in strong mobility", Proceedings of the 8th International Conference on Information Technology in Asia (CITA), Pages: 1 - 8, 2013.

[6] Seong-cheol Kim; Yeong-joon Kim, "EEP-MAC: An energy efficient priority-based MAC protocol for Wireless Sensor Networks", Proceedings of 2012 International Conference on ICT Convergence (ICTC), Pages: 591 592, 2012.

[7] Suraty Filho, Luiz Henrique ; Denis, Benoit ; Maman, Mickael, "Design and Analysis of Distributed Mobility Management Schemes for Wireless Sensor Networks", Proceedings of the 21th European Wireless Conference; European Wireless 2015, Page(s): 1 - 7, 20-22 May 2015.

[8] Mori, K. ; Div. of Electr. \& Electron. Eng., Mie Univ., Tsu, Japan ; Naito, K. ; Kaobayashi, H., "Active Period Reuse Mechanism for Autonomous Active Period Selection in Cluster-Tree Traffic Adaptive IEEE 802.15.4 WSNs with Cluster Mobility", 2014 IEEE 80th Vehicular Technology Conference (VTC Fall), Page(s): 1 - 5, 14-17 Sept. 2014.

[9] Al-Jemeli, M.; Hussin, F.A., "An Energy Efficient CrossLayer Network Operation Model for IEEE 802.15.4Based Mobile Wireless Sensor Networks", IEEE Sensors Journal, Volume: 15, Issue: 2, Pages: 684 - 692, 2015.

[10] Maha Bouaziza, Abderrezak Rachedib, "A Survey on Mobility Management Protocols in Wireless Sensor Networks based on 6LoWPAN Technology", Elsevier Computer Communications Journal, 22 October 2014.

[11] Al-Fuqaha, A.; Guizani, M.; Mohammadi, M.; Aledhari, M.; Ayyash, M., "Internet of Things: A Survey on Enabling Technologies, Protocols and Applications", IEEE Communications Surveys \& Tutorials, Volume: PP, Issue: 99, 2015.

[12] F. Teraoka, et all., "Unified Layer 2 (L2) Abstractions for Layer 3 (L3)-Driven Fast Handover", IETF, RFC 5184, May 2008. 